\title{
With no interaction, knockdown of Apollon and MDR1 reverse the multidrug resistance of human chronic myelogenous leukemia K562/ADM cells
}

\author{
JIE-RU CHEN $^{1 *}$, XIU-HONG JIA ${ }^{1 *}$, HONG WANG ${ }^{1}$, YING-JIE YI ${ }^{1}$ and YOU-JIE LI ${ }^{2}$ \\ ${ }^{1}$ Department of Pediatrics, The Affiliated Hospital of Binzhou Medical University, Binzhou, Shandong 256603; \\ ${ }^{2}$ Department of Biochemistry and Molecular Biology, Key Laboratory of Tumour Molecular Biology, \\ Binzhou Medical University, Yantai, Shandong 264003, P.R. China
}

Received August 7, 2016; Accepted September 22, 2016

DOI: 10.3892/or.2017.5535

\begin{abstract}
Chemotherapy is the main treatment method for patients with chronic myeloid leukemia (CML) and has achieved marked results. However, the acquisition of multidrug resistance (MDR) has seriously affected the quality of life and survival rate of patients. The overexpression of the inhibitors of apoptosis proteins (IAPs) and the adenosine triphosphate (ATP)dependent binding cassette $(\mathrm{ABC})$ transporters are the two main causes of MDR. Apollon and MDR1 are the most important and representative members, respectively, among the IAPs and ABC transporters. In the present study, we investigated the role of Apollon and MDR1 in chemotherapy resistance and their mechanism of interaction. We respectively knocked down the expression of Apollon and MDR1 using short hairpin RNA (shRNA) in adriamycin (ADM) resistant human CML K562 cells and examined the drug sensitivity, the consequences with regard to ADM accumulation and the alterations in the expression of Apollon and MDR1. The expression levels of Apollon and MDR1 mRNA were higher in the K562/ADM cells compared with the parental K562 cells as determined by reverse transcription-polymerase chain reaction (RT-PCR). The plasmids of Apollon and MDR1 shRNA were respectively stably transfected into K562/ADM cells using Lipofectamine 2000. The transfection efficiency was detected by fluorescence microscopy. Cell Counting Kit-8 (CCK-8) assay revealed that Apollon or MDR1 knockdown significantly increased the chemosensitivity of the K562/ADM cells to ADM. Flow cytometric assay revealed that K562/ADM/shMDR1 cells exhibited a significantly increased
\end{abstract}

Correspondence to: Dr Xiu-Hong Jia, Department of Pediatrics, The Affiliated Hospital of Binzhou Medical University, Binzhou, Shandong 256603, P.R. China

E-mail: jiaxiuhong001@163.com

${ }^{*}$ Contributed equally

Key words: chronic myelogenous leukemia, Apollon, MDR1, multidrug resistance, multidrug resistance reversal intracellular accumulation of ADM, and that changes were not found in the K562/ADM/shApollon cells. Compared with the parental K562/ADM cells, a significantly decreased expression of Apollon mRNA and protein was determined in the K562/ ADM/shApollon cells without affecting the expression of MDR1 as determined by RT-PCR and western blotting. Likewise, the expression levels of MDR1 mRNA and protein also markedly downregulated in the K562/ADM/shMDR1 cells had no effect on Apollon expression. Collectively, our findings demonstrated, for the first time, that downregulation of Apollon or MDR1 through stable transfection with the Apollon- or MDR1-targeting shRNA induced MDR reversal through respective inhibition of Apollon or MDR1 expression and function. However, the reversal mechanism of Apollon and MDR1 revealed no direct interaction with each other.

\section{Introduction}

Chronic myeloid leukemia (CML) is a clonal disease of hematopoietic stem cells characterized by a reciprocal translocation between chromosome 9 and 22 [t(9;22) (q34;q11)], an event that leads to the formation of the BCR-ABL oncogene which encodes the BCR-ABL oncogenic fusion protein with constitutive tyrosine kinase activity $(1,2)$. The incidence of CML is approximately one individual/100,000 population/year with a slight male predominance and rising incidence with age, accounting for $\sim 15 \%$ of newly diagnosed cases of leukemia in adults (1). Since characterized by the constitutively active BCR-ABL, an important understanding of the molecular pathogenesis of CML has afforded the potential for target-oriented therapy. As expected, the advent of tyrosine kinase inhibitors (TKIs) has drastically revolutionized the treatment prognosis of CML patients from a potentially fatal disorder to a disorder amenable simply with lifelong oral medication and compatible with a normal lifespan $(1,2)$. However, $\sim 10-15 \%$ of patients with CML remain resistant to TKIs and more than $62.5 \%$ of the patients may experience multidrug resistance (MDR) at risk of disease progression $(1,3)$. The acquisition of MDR has become a major clinical problem and continues to be a critical obstacle for chemotherapy regimen treatment in CML. Therefore, elucidation of the 
molecular mechanisms underlying cancer MDR has been performed extensively in order to identify putative targets in human cancers.

MDR is considered to possess a multifactorial phenotype and occurs as a result of two most crucial causes: the overexpression of the inhibitors of apoptosis proteins (IAPs) and cell membrane-bound adenosine triphosphate (ATP)binding cassette $(\mathrm{ABC})$ transporters (4-8). It has been recognized that resistance to apoptosis promotes the genesis and progression of cancer and helps cancer cells escape from chemotherapy-induced cytotoxicity, thus, causing cancer cell chemoresistance $(6,9,10)$. Apollon/BIR-containing protein 6 (BIRC6; also known as BRUCE) the largest and the only membrane-bound member of the IAP family, which is located on the outer membrane of the Golgi apparatus, plays an important role in cancer genesis and progression and is always abnormally overexpressed in a variety of human cancers $(9,11,12)$. ABC transporters which work as drug efflux pumps, play critical roles in human cancer cell resistance to chemotherapy drugs. Among these ABC transporters, multidrug resistance 1 [MDR1; also known as P-glycoprotein (P-gp) or ABCB1] is the most representative and relevant drug transporter and plays a critical role in human CML cell resistance to chemotherapy drugs $(4,8,13)$.

Although IAPs and P-gp are always overexpressed and coexist in multidrug resistant cancer cell lines $(5,14-16)$, the interrelationship between IAPs and ABC transporters is far from being completely understood. Clones of adriamycin (ADM) resistant CML K562/ADM cells were established by continuous exposure to ADM. Our previous study, demonstrated that the ADM-selected K562/ADM subline of our laboratory revealed a 31.78 -fold higher resistance to ADM when compared with the parental K562 cells (17). In the present study, we investigated the effect of short hairpin RNA (shRNA) knockdown of the Apollon or MDR1 gene combined with ADM on the chemosensitivity of the human CML cell line K562/ADM, and evaluated the possible relationship between Apollon and MDR1 expression and the targeted gene therapy response.

\section{Materials and methods}

Chemicals and reagents. ADM purchased from Melone Pharmaceutical Co., Ltd. (Dalian, China) was dissolved in $\mathrm{dd}_{2} \mathrm{O}$ at a concentration of $2 \mathrm{~g} / 1$ and stored at $-20^{\circ} \mathrm{C}$. Anti-Apollon/BIRC6, anti-MDR1/P-gp and anti-GADPH rabbit polyclonal antibodies $(\mathrm{pAb})$ were respectively obtained from Abcam Inc. (Cambridge, UK), Bioss Bio-Technology Co., Ltd. (Beijing, China) and Goodhere Biotechnology Co., Ltd. (Hangzhou, China).

Cell lines and cell culture. The human CML K562 cells were obtained from the Key Laboratory of Tumour Molecular Biology of Binzhou Medical University (Binzhou, China) and its MDR subline K562/ADM was obtained from the Department of Pharmacology at The Institute of Hematology of the Chinese Academy of Medical Sciences (Tianjin, China). The cells were maintained in RPMI-1640 medium supplemented with $10 \%$ fetal bovine serum (FBS; both from HyClone, Logan, UT, USA), $100 \mathrm{U} / \mathrm{ml}$ of penicillin and
$0.1 \mathrm{mg} / \mathrm{ml}$ streptomycin at $37^{\circ} \mathrm{C}$ in a humidified atmosphere containing $5 \% \mathrm{CO}_{2}$. $\mathrm{K} 562 / \mathrm{ADM}$ cells were cultured in the same medium with an additional $4 \mathrm{mg} / \mathrm{l}$ ADM. Before the experiment, K562/ADM cells were cultured in drug-free medium for $72 \mathrm{~h}$.

Determination of gene expression by reverse transcription-polymerase chain reaction (RT-PCR). Approximately $3 \times 10^{6}$ cells were harvested for RT-PCR analysis. Total RNA was isolated from the cells using TRIzol reagent (Invitrogen, Carlsbad, CA, USA) according to the manufacturer's instructions. Total RNA was reverse transcribed to cDNA and stored at $-20^{\circ} \mathrm{C}$. Primers were designed using Primer 5 version 5.6.0 software and synthesized by Sangon Biotech Co., Ltd. (Shanghai, China). Primers used in RT-PCR were as follows: Apollon forward, 5'-TGGCTCAAGCTGGATTTTAT-3' and reverse, 5'-TTCAGACCAAGGTTCATCAG-3'; MDR1 forward, 5'-GGAGCCTACTTGGTGGCACATAA-3' and reverse, 5'-TGGCATAGTCAGGAGCAAATGAAC-3'; GAPDH forward, 5'-GAAGGTGAAGGTCGGAGTC-3' and reverse, 5'-GAAGATGGTGATGGGATTTC-3'. RT-PCR was performed on an ABI Prism 7500 Real-Time PCR system (Applied Biosystems, Foster City, CA, USA) using a SYBR-Green Reaction kit (Takara Bio, Otsu, Shiga, Japan). The reaction system of the PCR was: SYBR-Green reagent, forward and reverse primer, template cDNA and nuclease-free distilled water. PCR programs were carried out as follows: $95^{\circ} \mathrm{C}$ for $30 \mathrm{sec}$, followed by 45 cycles at $95^{\circ} \mathrm{C}$ for $5 \mathrm{sec}$ and $60^{\circ} \mathrm{C}$ for $30 \mathrm{sec}$. GAPDH served as an internal control. The PCR products were separated by $1 \%$ agarose gels (Takara Biotechnology Co., Ltd., Dalian, China) and stained with ethidium bromide for $15 \mathrm{~min}$. The gels were scanned and analyzed by the Tanon Gel Imaging system. RT-PCR for each gene of each cDNA sample was assayed in triplicate.

Plasmid construction and stable transfection of cells. The Apollon-targeted shRNA (termed as shApollon), MDR1-targeted shRNA (termed as shMDR1) and the negative control shRNA (termed as shControl) (Table I) were synthesized by recombinant plasmids containing the green fluorescent protein (GFP) vector, pGPH1, purchased from Shanghai GenePharma Co., Ltd. (Shanghai, China) after being chemically synthesized, annealed, desalted and purified. The shApollon and shMDR1 sequences were selected according to a previous study in our laboratory and the research was performed by Du et al, respectively $(18,19)$. Both of the constructs were confirmed by sequence analysis.

Prior to transfection, K562/ADM cells were seeded into 6-well plates and cultured for $12 \mathrm{~h}$ in serum-free RPMI-1640 medium and antibiotics to $60-80 \%$ confluency. Transfection was performed at a final concentration of $1 \mu \mathrm{g}$ of plasmid-expressing shRNA using Lipofectamine 2000 transfection reagent (Invitrogen) according to the manufacturer's instructions. Six hours after transfection, RPMI-1640 medium containing 10\% FBS was added, and the cells were then incubated under the aforementioned conditions. G418 (500-1,000 ng/ml; Sigma, St. Louis, MO, USA) was then added to the medium after $24 \mathrm{~h}$ of transfection, and the cells were cultured for 1 month to permit selection. Stable cells were assessed by fluorescence microscopy (Olympus DP71; Olympus, Tokyo, Japan), and analyzed using 
Table I. Synthetic Apollon and MDR1 shRNA sequence.

Genes

Forward and reverse primers

\begin{tabular}{lll}
\hline Apollon & F & 5'-CUGCCUCUUUCAGGCAAUATT-3' \\
& $\mathrm{R}$ & 5'-UAUUGCCUGAAAGAGGCAGTT-3' \\
MDR1 & $\mathrm{F}$ & 5'-GATCCCGAAACCAACTGTCAGTGTATCAAGAGTACACTGACAGTTGGTTTCTTTTTT-3' \\
& $\mathrm{R}$ & 5'-CTAGAAAAAAGAAACCAACTGTCAGTGTACTCTTGATACACTGACAGTTGGTTTCGG-3' \\
Control & F & 5'-GTTCTCCGAACGTGTCACGTCAAG-3' \\
& $\mathrm{R}$ & 5'-GATTACGTGACACGTTCGGAGAATT-3'
\end{tabular}

MDR1, multidrug resistance 1; shRNA, short hairpin RNA; F, forward; R, reverse.

RT-PCR and western blotting. GFP expression was considered as an indicator of efficiency of gene delivery. K562/ADM cells were divided into 4 groups: group 1 (K562/ADM, untransfected cells); group 2 (K562/ADM/shControl, pGPH1-GFP-NeoNC-transfected cells); group 3 (K562/ADM/shApollon, pGPH1-GFP-Neo-Apollon-transfected cells); and group 4 (K562/ADM/shMDR1, pGPH1-GFP-Neo-MDR1 transfected cells).

Cytotoxicity assays. Cell Counting Kit-8 (CCK-8; Dojindo Molecular Technologies, Inc., Shanghai, China) was used to determine the survival rate of all the cell groups incubated with ADM. Firstly, the cells were seeded in a 96-well plate at a density of $5 \times 10^{3}$ cells/well in RPMI-1640 medium containing $10 \%$ FBS. Then, various concentrations of ADM at 4-64 mg/l were added to each well, accordingly. After the cells were incubated at $37^{\circ} \mathrm{C}$ in $5 \% \mathrm{CO}_{2}$ for $24 \mathrm{~h}, 10 \mu \mathrm{lCCK}-8$ solution was added to each well and incubation followed for $4 \mathrm{~h}$. Subsequently, the absorbance was assessed at $490 \mathrm{~nm}$ with a fluorescence spectrofluorometer (F-7000; Hitachi High-Technologies Corporation, Tokyo, Japan). A blank well containing only medium and drugs, was used as a control. The $50 \%$ inhibition of cell growth $\left(\mathrm{IC}_{50}\right)$ produced by $\mathrm{ADM}$ was calculated using the untreated cells as the $100 \%$ viable control. The reversal fold (RF) values, as potency of reversal, were obtained from the following formula: $\mathrm{RF}=\mathrm{IC}_{50}$ of $\mathrm{ADM}$ in untransfected cells/ $\mathrm{IC}_{50}$ of $\mathrm{ADM}$ in the differently transfected cell groups.

Cellular uptake of ADM. All cell groups were plated in 6-well plates at a concentration of $1 \times 10^{6}$ cells in $1 \mathrm{ml}$ of growth medium. After incubation at $37^{\circ} \mathrm{C}$ for $24 \mathrm{~h}, 2 \mathrm{mg} / 1$ of $\mathrm{ADM}$ was added for another $1 \mathrm{~h}$ of incubation at $37^{\circ} \mathrm{C}$. Cells without ADM were used to evaluate cell auto-fluorescence. Then, the cells were harvested by centrifugation and washed twice with ice-cold phosphate-buffered saline (PBS). The cell-associated mean fluorescence intensity (MFI) of ADM was detected by flow cytometer using a FACSCalibur (Beckman Coulter, Brea, CA, USA) with excitation/emission wavelengths at 485/580 nm.

Western blotting. The cells were harvested and washed with PBS. Lysis buffer (100 $\mu \mathrm{l})$ was added and the protein concentration of the lysate was determined using a Bicinchoninic Acid Protein Assay kit (both from Beyotime Biotechnology, Shanghai, China). The lysed samples containing $50 \mu \mathrm{g}$ of protein were separated by $6-8 \%$ sodium dodecyl sulphate-polyacrylamide gel electrophoresis (SDS-PAGE; Beyotime Biotechnology) with a constant voltage of $80 \mathrm{~V}$ for $0.5 \mathrm{~h}$ and $120 \mathrm{~V}$ for another $1.5 \mathrm{~h}$. The resolved proteins were electrophoretically transferred to polyvinylidine difluoride membranes (EMD Millipore, Bedford, MA, USA) and blocked with $5 \%$ skimmed milk for $2 \mathrm{~h}$. Subsequently, the membranes were incubated overnight at $4{ }^{\circ} \mathrm{C}$ with specific primary rabbit polyclonal antibodies against Apollon (1:1,000), P-gp (1:500) and GADPH $(1: 1,000)$. The following day, the membranes were incubated in horseradish peroxidase-labeled goat anti-rabbit immunoglobulin $\mathrm{G}(1: 5,000)$ for $2 \mathrm{~h}$ at room temperature. Finally, images were captured using a FluorChem FC2 Gel Imaging System (Alpha Innotech, San Leandro, CA, USA). The intensity of each band was normalized to that of GADPH for their respective lanes.

Statistical analysis. Statistical analyses were performed using SPSS 17. 0 software (IBM SPSS, Armonk, NY, USA). Data are expressed as the means \pm SD. Statistical comparisons were evaluated by one-way ANOVA. Values of $\mathrm{P}<0.05$ were considered to indicate a statistically significant result.

\section{Results}

K562/ADM cells overexpress Apollon and MDR1 in comparison with the parental sensitive cells. RT-PCR analysis was performed to determine the expression of Apollon and MDR1 in parental K562 cells, and the resistant K562/ADM cells. As shown in Fig. 1, our results demonstrated that both the K562 and K562/ADM cells exhibited high expression levels of Apollon and MDR1, but that the K562/ADM cells expressed much higher levels of Apollon and MDR1 than the K562 cells $(\mathrm{P}<0.05)$. Overall, our results revealed that in contrast to parental sensitive K562 cells, MDR K562/ADM cells exhibited a higher level of Apollon/MDR1 co-expression, which may give rise to MDR.

Transfection efficiency of the recombinant plasmids in the K562/ADM cells. Fluorescence was detected using a fluorescence microscope after G418 selection. The expression of green fluorescent protein was detected in most of the stablytransfected cells (>90\%), indicating a very high transfection efficiency (Fig. 2). These results indicated that the K562/ADM cells were successfully transfected with the plasmids. 

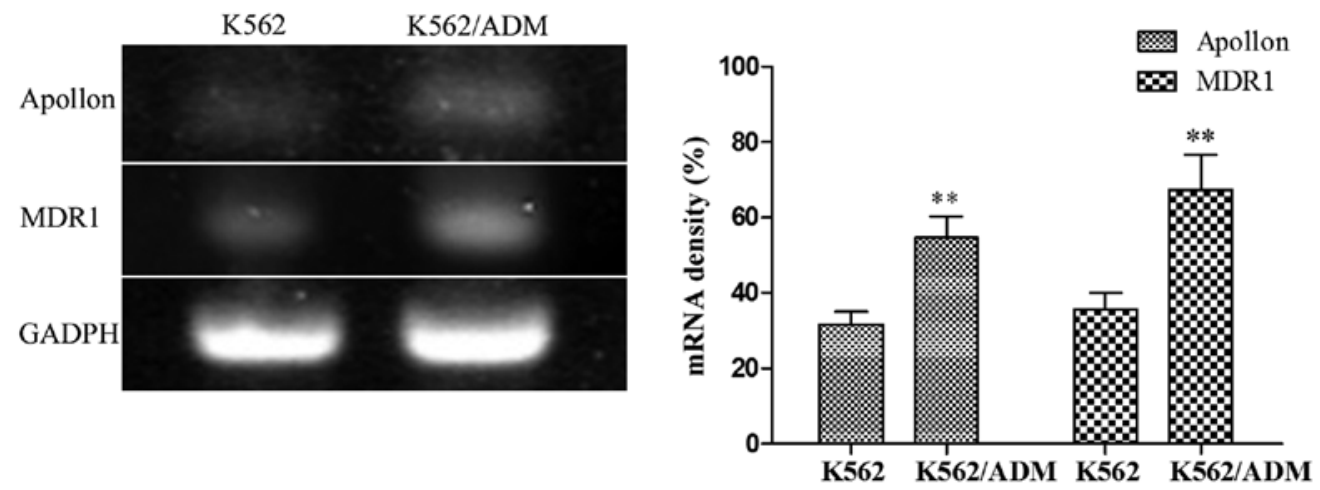

Figure 1. Analysis of Apollon and MDR1 expression in parental and ADM-resistant K562 cells by RT-PCR. The expression of Apollon and MDR1 were normalized to GADPH revealing a stronger expression in the K562/ADM cells than the parental cells. The results are represented as the mean \pm SD of triplicate experiments; ${ }^{* *} \mathrm{P}<0.01$ vs. the parental cells. MDR1, multidrug resistance protein 1; ADM, adriamycin; RT-PCR, reverse transcription-polymerase chain reaction.

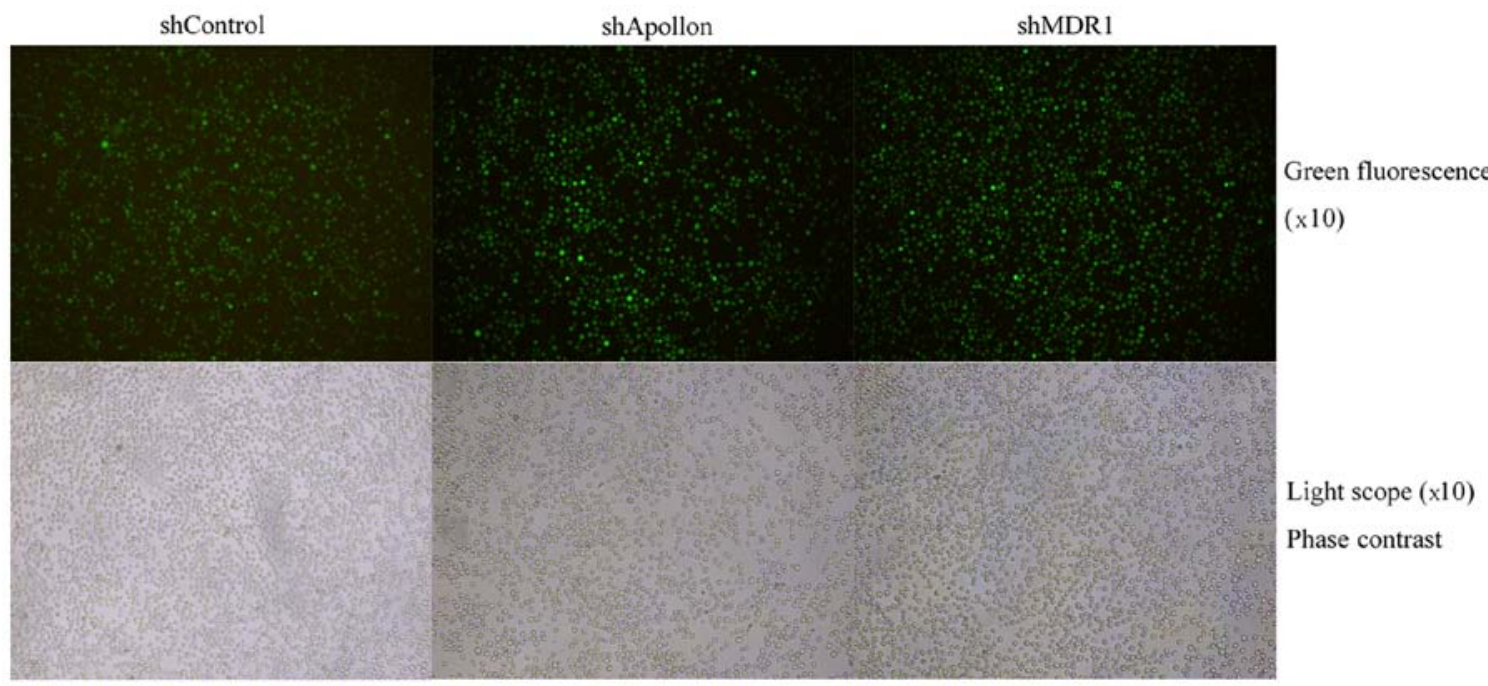

Figure 2. K562/ADM cells were stably transfected with negative control shRNA (shControl), Apollon knockdown shRNA (shApollon) and MDR1 knockdown shRNA (shMDR1), respectively. The transfection efficiency was detected by fluorescence microscopy. The average transfection efficiency of each group was $>90 \%$. MDR1, multidrug resistance protein $1 ;$ ADM, adriamycin.

Apollon and MDR1 knockdown increases the chemosensitivity of K562/ADM cells to ADM. We examined whether Apollon or MDR1 knockdown sensitizes CML K562/ADM cells to ADM. The CCK-8 assay detected the growth inhibition rate of cells after exposure to a range of concentrations of ADM for $24 \mathrm{~h}$. As shown in Fig. 3, the K562/ADM/shApollon and K562/ADM/shMDR1 cells exhibited significantly greater growth inhibition than the K562/ADM/shControl and the untransfected K562/ADM cells by ADM. Then, the $\mathrm{IC}_{50}$ values of ADM were calculated by CCK-8 assay data to determine whether downregulation of Apollon and MDR1 contributed to drug sensitivity in the K562/ADM cells. The $\mathrm{IC}_{50}$ values of $\mathrm{ADM}$ in the K562/ADM cells were $32.60 \pm 3.215$, 30.18 \pm 4.03 , $17.53 \pm 1.595$ and $5.785 \pm 1.8895 \mathrm{mg} / \mathrm{l}$ for the ADM alone, shControl, shApollon and shMDR1 groups, respectively. The reversal fold-change of shApollon and shMDR1 was 1.86 and 5.64 , respectively (Table II; $\mathrm{P}<0.05$ ). Therefore, the downregulation of Apollon and MDR1 increased the sensitivity of K562/ADM cells to ADM.

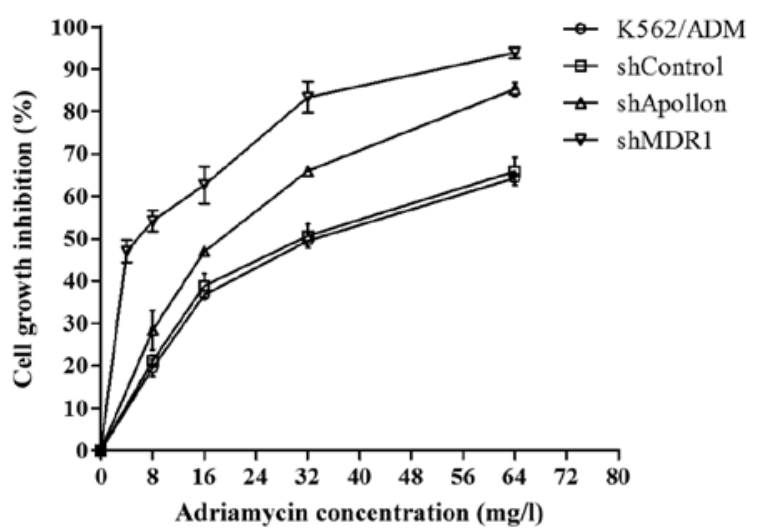

Figure 3. Apollon and MDR1 knockdown alters ADM-induced cytotoxicity of K562/ADM cells. Growth curves were based on data from the CCK-8 assays in all cell groups: untransfected K562/ADM, K562/ADM/shControl, K562/ADM/shApollon and K562/ADM/shMDR1 cells, following $24 \mathrm{~h}$ of treatment with various concentrations of ADM. A significant increased drug sensitivity was noted in the K562/ADM/shApollon and K562/ADM/shMDR1 cells. Results are expressed as the mean \pm SD of 3 independent experiments. MDR1, multidrug resistance protein 1; ADM, adriamycin. 
A
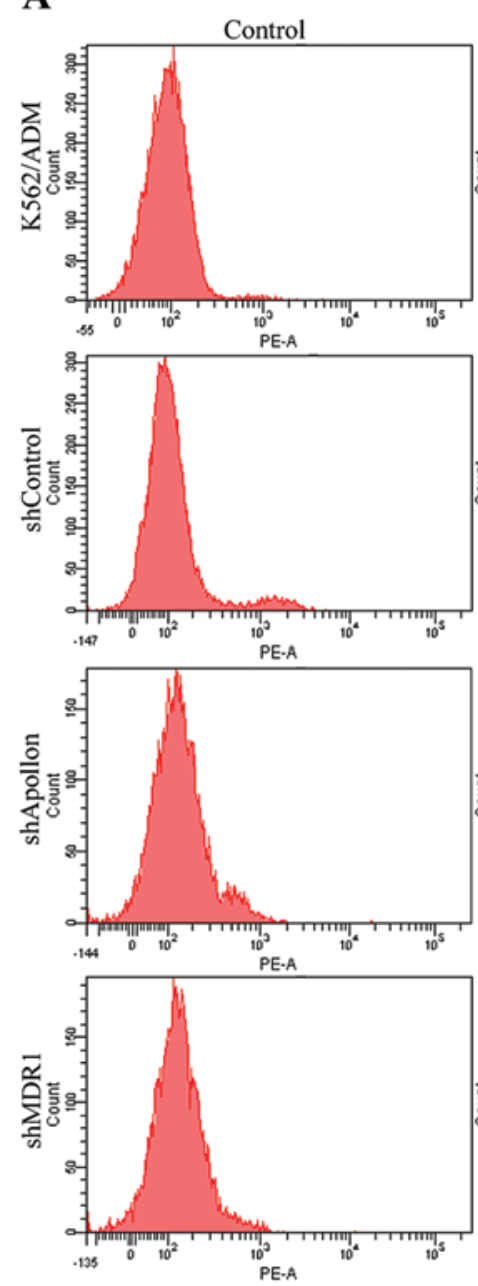
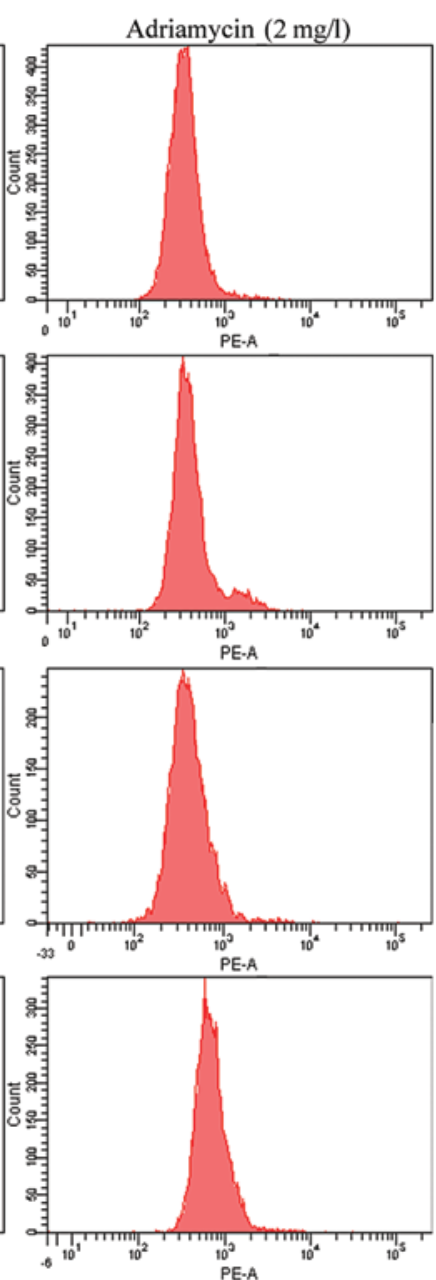

B

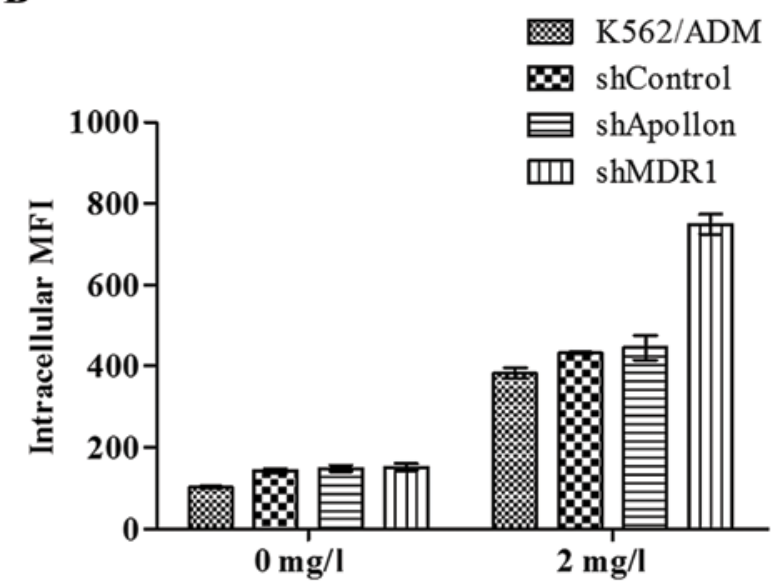

ADM concentration

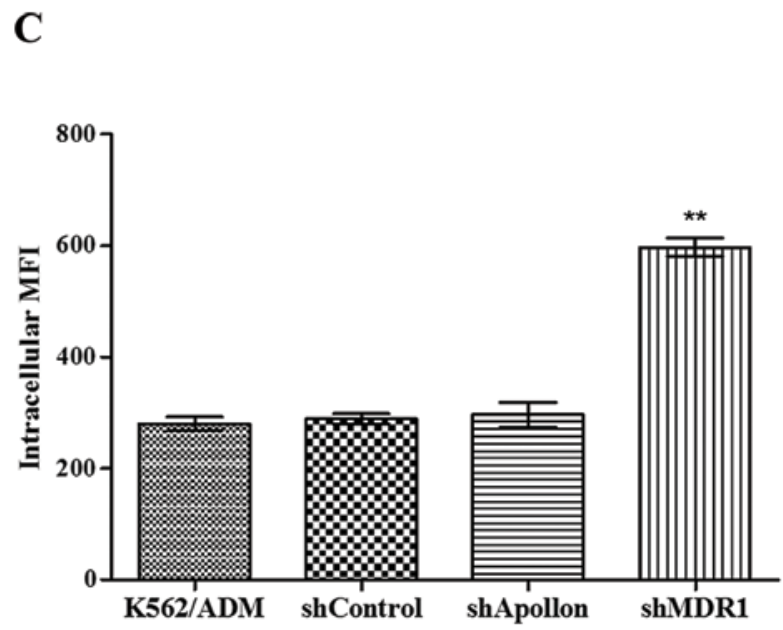

Figure 4. Effect of Apollon and MDR1 knockdown on the intracellular accumulation of ADM in K562/ADM cells. (A) All cell groups were treated with or without ADM ( $2 \mathrm{mg} / \mathrm{l})$ for $1 \mathrm{~h}$ and the fluorescence of ADM in cells was assessed by FCM. (B) Analysis of auto-fluorescence and the MFI of ADM in all cell groups. (C) After standardization, the FCM results revealed that there was a significant increase in intracellular accumulation of ADM only determined in the K562/ADM/shMDR1 cells. The other cell groups did not exhibit this change. The bars represent the means \pm SD; $n=4 ; \mathrm{P}>0.05$, compared to the untransfected K562/ADM cell group; ${ }^{* *} \mathrm{P}<0.01$ compared to the untransfected K562/ADM cell group. MDR1, multidrug resistance protein 1; ADM, adriamycin; FCM, flow cytometric assay; MFI, mean fluorescence intensity.

Table II. Effect of Apollon and MDR1 knockdown on the sensitivity of K562/ADM cells towards ADM by CCK-8 assay.

\begin{tabular}{lll}
\hline Treatment & $\mathrm{IC}_{50}(\mu \mathrm{g} / \mathrm{ml})$ & $\mathrm{RF}$ \\
\hline ADM alone & $32.60 \pm 3.215$ & \\
shControl & $30.18 \pm 4.03$ & 1.08 \\
shApollon & $17.53 \pm 1.595^{\mathrm{a}}$ & 1.86 \\
shMDR1 & $5.785 \pm 1.8895^{\mathrm{a}}$ & 5.64 \\
\hline
\end{tabular}

${ }^{\mathrm{a}} \mathrm{P}<0.05$ vs. ADM alone. MDR1, multidrug resistance 1; ADM, adriamycin; CCK-8, Counting Kit-8; RF, reversal fold. Results are expressed as the means $\pm \mathrm{SD}$ of triplicate experiments.

Effect of Apollon and MDRl knockdown on the intracellular accumulation of ADM. The intracellular accumulation of ADM was previously found to decrease significantly in
K562/ADM cells compared to parental K562 cells (20). Thus, we examined whether knockdown of Apollon and MDR1 increases the intracellular accumulation of ADM in K562/ ADM cells. As shown in Fig. 4, our results demonstrated that the K562/ADM/shMDR1 cells had an increased intracellular accumulation of ADM $(\mathrm{P}<0.05)$ while the other cell groups did not exhibit this change. Thus, our result indicated that shMDR1 significantly inhibited the expression and function of P-gp and that the knockdown of Apollon did not affect the function of P-gp.

Apollon knockdown shRNA significantly decreases the expression level of Apollon in K562/ADM/shApollon cells, but has no impact on MDR1 expression. As Apollon and MDR1 were co-expressed and at high levels in the CML K562/ADM cells, we explored whether knockdown of Apollon affects MDR1 expression. After stable transfection with plasmids shControl and shApollon, we used RT-PCR and western blot assays to confirm the knockdown efficiency on Apollon mRNA and 
A

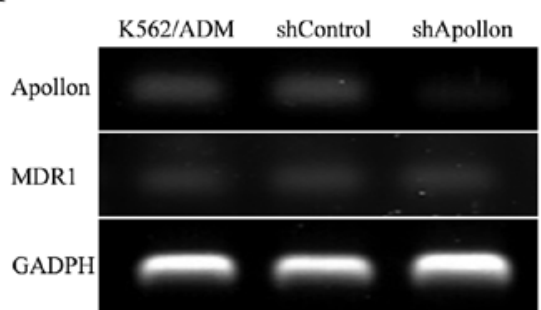

B

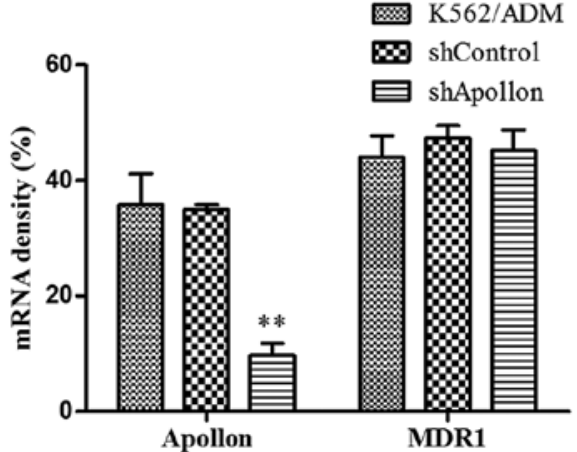

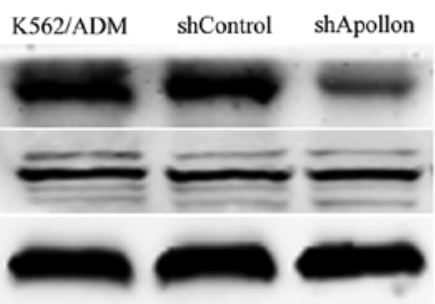

D

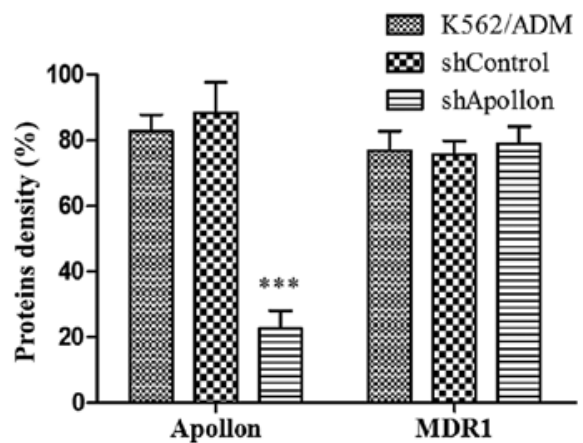

Figure 5. Apollon knockdown significantly decreases Apollon expression at both the mRNA and protein levels in K562/ADM/shApollon cells with no effect on the expression of MDR1. The Apollon and MDR1 mRNA and protein expression levels were determined by RT-PCR and western blotting, respectively. (A) Image of agarose ethidium bromide gel. (B) Analysis of Apollon and MDR1 mRNA expression. (C) Image of SDS-PAGE. (D) Analysis of Apollon and P-gp protein expression. The expression of Apollon and MDR1 were calculated after normalization to GADPH expression. Results are expressed as the mean $\pm \mathrm{SD}$ of triplicate experiments. Bars represent the means $\pm \mathrm{SD} ; \mathrm{n}=3 ; \mathrm{P}>0.05$, compared to the untransfected K562/ADM cell group; ${ }^{* *} \mathrm{P}<0.01,{ }^{* * * *} \mathrm{P}<0.001$ vs. the untransfected K562/ADM cell group. MDR1, multidrug resistance protein 1; ADM, adriamycin.

A

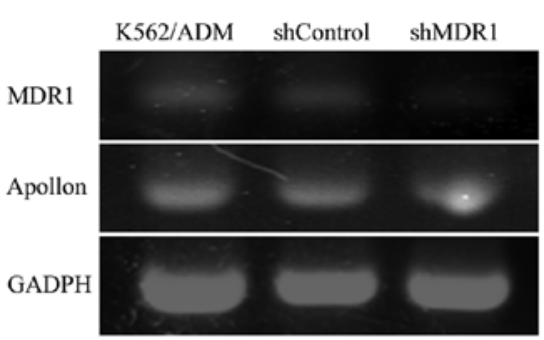

B

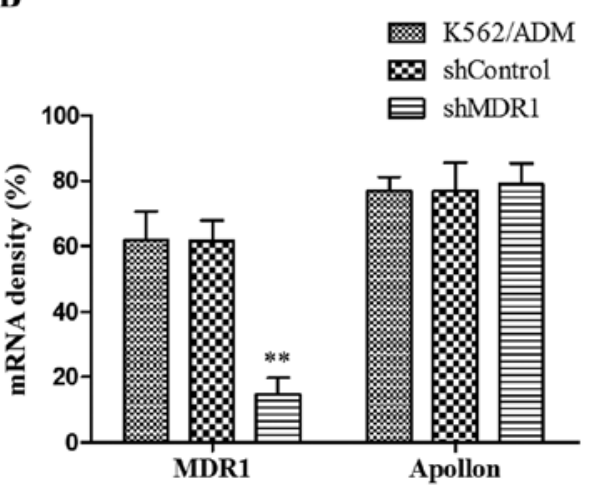

C

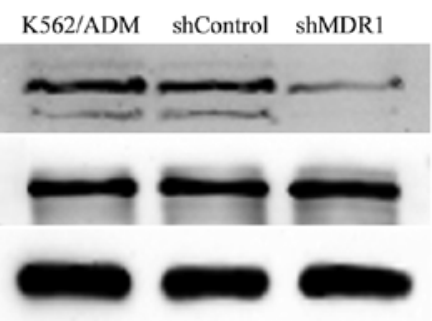

D

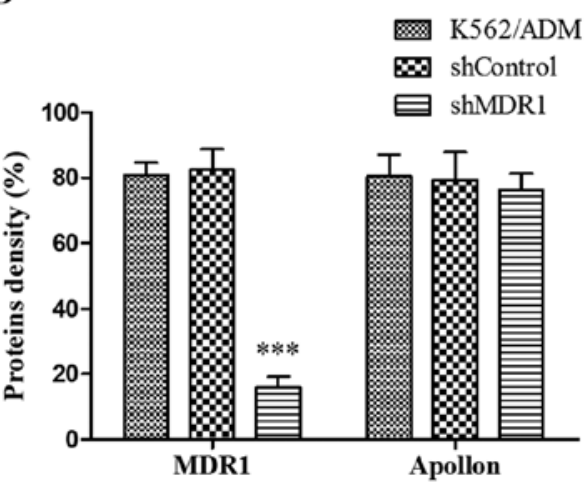

Figure 6. MDR1 silencing significantly downregulates the expression of MDR1 at both the mRNA and protein levels in K562/ADM/shMDR1 cells, but has no effect on Apollon expression. The MDR1 and Apollon mRNA and protein expression levels were determined by RT-PCR and western blotting, respectively. (A) Image of agarose ethidium bromide gel. (B) Analysis of MDR1 and Apollon mRNA expression. (C) Image of SDS-PAGE. (D) Analysis of P-gp and Apollon protein expression. GADPH was used as an internal control. The results are expressed as the mean \pm SD for 3 independent experiments. The bars represent the means $\pm \mathrm{SD} ; \mathrm{n}=3 ; \mathrm{P}>0.05$, compared to the untransfected K562/ADM cell group; ${ }^{* *} \mathrm{P}<0.01,{ }^{* * * *} \mathrm{P}<0.001$ compared to the untransfected K562/ADM cell group. MDR1, multidrug resistance protein 1; ADM, adriamycin.

protein levels and the expression change in MDR1. As shown in Fig. 5, compared with the untransfected K562/ADM cells and the K562/ADM/shControl cells, the levels of Apollon mRNA and protein in the K562/ADM/shApollon cells were 
significantly decreased $(\mathrm{P}<0.05)$. However, the levels of MDR1 mRNA and protein in the K562/ADM/shApollon cells were not altered.

MDRI knockdown shRNA efficiently downregulates the expression of MDR1 in K562/ADM/shMDR1 cells but has no affect on the expression of Apollon. Likewise, to explore whether knockdown of MDR1 affects the expression of Apollon, after stable transfection with the plasmids shControl and shMDR1, we investigated the expression changes in MDR1 and Apollon by RT-PCR and western blot assays. As shown in Fig. 6, the K562/ADM/shMDR1 cells exhibited significantly decreased mRNA and protein levels of MDR1 and unchanged expression of Apollon. Collectively, our experiments revealed that silencing of Apollon or MDR1 respectively, did not affect the expression of the other, and that the reversal effect of Apollon and MDR1 knockdown in CML K562/ADM cells was mainly dependent on the downregulation of the targeted gene expression.

\section{Discussion}

The constitutive tyrosine kinase (TK) activity of the BCR-ABL oncoprotein in CML plays an important role in promoting cell proliferation and survival, which provides an understanding of the pathogenesis of CML and affords a target-oriented therapy. Although targeted BCR-ABL tyrosine kinase inhibitors (TKIs) significantly improve the prognosis of CML patients, the development of MDR becomes the most serious clinical problem responsible for the failure of chemotherapy. There is a growing concern in understanding the mechanisms of MDR and finding new target-oriented therapies to overcome drug resistance.

The cause of MDR is often multifactorial. However, the most widely studied and important mechanism is the overexpression of $\mathrm{ABC}$ transporters. MDR1, also known as P-glycoprotein (P-gp) or ABCB1, is the first identified member of the $\mathrm{ABC}$ transporter family, mainly expressed in the plasma membrane that pumps cytotoxic drugs out of the cells through active transportation (21). There is an increasing amount of preclinical data supporting that MDR1 is one of the most important and representative $\mathrm{ABC}$ transporters and plays a crucial role in MDR of human CML. MDR1 is always overexpressed and abnormally activated in cancer cells and the expression level of MDR1 is correlated with MDR (8). Moreover, it has been reported that the overexpression of MDR1 may be a risk factor for the treatment and prognosis of CML (22). Furthermore, studies have shown that the genetic polymorphisms of MDR1 predict the clinical response of CML patients to TKIs (23-25). Numerous studies revealed that downregulating the expression of P-gp restored drug sensitivity $(3,6,7,17,19,20)$. Thus, MDR1 plays a crucial role in human CML cell resistance to chemotherapy drugs.

Meanwhile, another important cause for the acquisition of MDR is apoptosis inhibition. Apoptosis is the process of cell self-destruction during physiologic or pathologic conditions under the control of genes, which plays a central role in the development and homeostasis of multicellular organisms. The ability to evade apoptosis is considered to be a major cause of tumor resistance to chemotherapy since chemo- therapeutic agents mainly act depending on the induction of apoptosis (11). The inhibitors of apoptosis proteins (IAPs) play a strong antagonistic role to cell apoptosis and potentially contribute to oncogenesis and resistance to antitumor treatment $(6,9)$. Increased IAP expression has been found in a variety of human cancers, including CML (4-6,9-12,14-16). Apollon is the largest and the only membrane-bound member of the IAP family. Previous studies have reported that overexpression of the IAP, Apollon, is associated with unfavorable clinical features and negatively impacts relapse-free survival in childhood acute leukemia (AL) $(26,27)$. Our previous study demonstrated that Apollon is constitutively expressed in CML K562 cells and knockdown of Apollon by short hairpin RNA (shRNA) significantly enhanced K562 cell response to cytotoxic drugs (18).

Since IAPs and ABC drug transporters are the two most important causes of MDR, the interactions between IAPs and $\mathrm{ABC}$ transporters have been extensively investigated in recent years. It has been reported that IAPs are always co-expressed with MDR1 at high levels in a variety of human cancers, and that some members of the IAP family have a positive correlation with MDR1. The expression of several IAPs could even be modulated by MDR1 $(4-6,15,16)$. However, some researchers have reported that the overexpression of survivin and XIAP in MDR cancer cells is not directly related to MDR1 (14). Even so, the relationship between Apollon and MDR1 in MDR cancer cells remains unclear.

In the present study, we designed and screened shRNA molecules, respectively targeting Apollon and MDR1 genes to investigate the role of Apollon and MDR1 in chemotherapy resistance and their mechanism of interaction in CML K562/ADM cells. Firstly, we determined that the expression of Apollon and MDR1 in K562/ADM cells was significantly higher than in the parental K562 cells. Then, we respectively stably transfected the targeting shRNAs of Apollon and MDR1 in K562/ADM cells to block gene expression. We found that both Apollon and MDR1 knockdown significantly restored the sensitivity of ADM in K562/ADM cells. Then, our data demonstrated that MDR1 knockdown by shRNA significantly inhibited the expression and function of P-gp with increased intracellular accumulation of ADM. However, the intracellular accumulation of ADM did not change significantly in the Apollon-knockdown K562/ADM cells, which indicates that the downregulation of Apollon did not affect the function of P-gp. Additionally, the Apollon-targeting shRNA transfection significantly decreased the expression of Apollon without affecting the expression of MDR1. Similarly, we found that the expression of MDR1 was potently inhibited in MDR1-targeting shRNA transfected K562/ADM cells which did not exhibit any effect on Apollon expression. Therefore, it is reasonable to believe that Apollon and MDR1 play an important role in the chemotherapy resistance of CML K562/ADM cells although they have no direct interaction.

It has been revealed that Apollon and MDR1 both contribute to chemotherapy resistance, but they do not directly interact with each other. The shRNAs targeting Apollon and MDR1 significantly inhibited the transcription, translation and function of the targeted genes thereby enhancing the sensitivity of CML K562/ADM cells to ADM. These studies provide evidence in support of using shRNAs as a molecularly defined 
therapeutic approach for MDR in the treatment of CML and further studies are warranted to understand the mechanism of MDR in CML cells.

\section{Acknowledgements}

The present study was supported by the Shandong Science and Technology Committee (no. 2010GSF10264), the Foundation of Shandong Educational Committee (nos. J10LC60 and J11LC01), the Natural Science Foundation of Shandong Province (no. ZR2014HQ079), and the Projects of Medical and Health Technology Development Program in Shandong Province (no. 2014WS0183).

\section{References}

1. Apperley JF: Chronic myeloid leukaemia. Lancet 385: 1447-1459, 2015.

2. Jabbour E: Chronic myeloid leukemia: First-line drug of choice. Am J Hematol 91: 59-66, 2016.

3. Zhang X, Dong W, Zhou H, Li H, Wang N, Miao X and Jia L: $\alpha-2,8$-Sialyltransferase is involved in the development of multidrug resistance via PI3K/Akt pathway in human chronic myeloid leukemia. IUBMB Life 67: 77-87, 2015.

4. Hu M, Liu Y, Deng C, Han R, Jia Y, Liu S, Jiang Z, Cao X, $\mathrm{He} \mathrm{L}$ and Zhang Q: Enhanced invasiveness in multidrug resistant leukemic cells is associated with overexpression of P-glycoprotein and cellular inhibitor of apoptosis protein. Leuk Lymphoma 52: 1302-1311, 2011.

5. Liu F, Liu S, He S, Xie Z,Zu X and Jiang Y: Survivin transcription is associated with P-glycoprotein/MDR1 overexpression in the multidrug resistance of MCF-7 breast cancer cells. Oncol Rep 23: $1469-1475,2010$.

6. Notarbartolo M, Cervello M, Dusonchet L, Cusimano A and D'Alessandro N: Resistance to diverse apoptotic triggers in multidrug resistant HL60 cells and its possible relationship to the expression of P-glycoprotein, Fas and of the novel anti-apoptosis factors IAP (inhibitory of apoptosis proteins). Cancer Lett 180: 91-101, 2002

7. Kachalaki S, Baradaran B, Majidi J, Yousefi M, Shanehbandi D, Mohammadinejad S and Mansoori B: Reversal of chemoresistance with small interference RNA (siRNA) in etoposide resistant acute myeloid leukemia cells (HL-60). Biomed Pharmacother 75: 100-104, 2015.

8. Arrigoni E, Galimberti S, Petrini M, Danesi R and Di Paolo A: ATP-binding cassette transmembrane transporters and their epigenetic control in cancer: An overview. Expert Opin Drug Metab Toxicol 12: 1419-1432, 2016.

9. Saleem M, Qadir MI, Perveen N, Ahmad B, Saleem U, Irshad T and Ahmad B: Inhibitors of apoptotic proteins: New targets for anticancer therapy. Chem Biol Drug Des 82: 243-251, 2013.

10. Notarbartolo M, Cervello M, Poma P, Dusonchet L, Meli M and D'Alessandro N: Expression of the IAPs in multidrug resistant tumor cells. Oncol Rep 11: 133-136, 2004.

11. Zhang S, Tang WQ, Weng SQ, Liu XJ, Rao BQ, G u JX, Chen S, Wang Q, Shen XZ, Xue RY, Dong L: Apollon modulates chemosensitivity in human esophageal squamous cell carcinoma. Oncotarget5 (16): 7183-97, 2014

12. Chen Z, Naito M, Hori S, Mashima T, Yamori T and Tsuruo T: A human IAP-family gene, Apollon, expressed in human brain cancer cells. Biochem Biophys Res Commun 264: 847-854, 1999.
13. Peng XX, Tiwari AK, Wu HC and Chen ZS: Overexpression of P-glycoprotein induces acquired resistance to imatinib in chronic myelogenous leukemia cells. Chin J Cancer 31: 110-118, 2012.

14. Shi Z, Liang YJ, Chen ZS, Wang XH, Ding Y, Chen LM and Fu LW: Overexpression of Survivin and XIAP in MDR cancer cells unrelated to P-glycoprotein. Oncol Rep 17: 969-976, 2007.

15. Reis FR, Vasconcelos FC, Pereira DL, Moellman-Coelho A, Silva KL and Maia RC: Survivin and P-glycoprotein are associated and highly expressed in late phase chronic myeloid leukemia. Oncol Rep 26: 471-478, 2011.

16. Silva KL, de Souza PS, Nestal de Moraes G, MoellmannCoelho A, Vasconcelos FC and Maia RC: XIAP and $\mathrm{P}$-glycoprotein co-expression is related to imatinib resistance in chronic myeloid leukemia cells. Leuk Res 37: 1350-1358, 2013.

17. Chen JR, Jia XH, Wang H, Yi YJ, Wang JY and Li YJ: Timosaponin A-III reverses multi-drug resistance in human chronic myelogenous leukemia K562/ADM cells via downregulation of MDR1 and MRP1 expression by inhibiting PI3K/Akt signaling pathway. Int J Oncol 48: 2063-2070, 2016.

18. Jia XH, Xiao FF and Li JC: Effect of Apollon siRNA combined with tetramethylpyrazine on proliferation and apoptosis of leukemia K562 cells. Zhongguo Dang Dai Er Ke Za Zhi 16: 135-140, 2014 (In Chinese).

19. Du WT, Liu B, Gu DS, Han ZB, Liu PX, Xu J, Liang L, Zhao HF, Lu SH and Yang RC: Reversal of leukemia multidrug resistance by sequence-specific short hairpin RNA. Zhongguo Shi Yan Xue Ye Xue Za Zhi 17: 563-567, 2009 (In Chinese).

20. Wang L, Meng Q, Wang C, Liu Q, Peng J, Huo X, Sun H, Ma X and Liu K: Dioscin restores the activity of the anticancer agent adriamycin in multidrug-resistant human leukemia K562/adriamycin cells by down-regulating MDR1 via a mechanism involving NF- $\kappa$ B signaling inhibition. J Nat Prod 76: 909-914, 2013.

21. Sarkadi B,Homolya L, Szakács G and Váradi A: Human multidrug resistance $\mathrm{ABCB}$ and $\mathrm{ABCG}$ transporters: Participation in a chemoimmunity defense system. Physiol Rev 86: 1179-1236, 2006.

22. Agrawal M, Hanfstein B, Erben P, Wolf D, Ernst T, Fabarius A, Saussele S, Purkayastha D, Woodman RC, Hofmann WK, et al: MDR1 expression predicts outcome of $\mathrm{Ph}^{+}$chronic phase CML patients on second-line nilotinib therapy after imatinib failure. Leukemia 28: 1478-1485, 2014.

23. Zu B, Li Y, Wang X, He D, Huang Z and Feng W: MDRl gene polymorphisms and imatinib response in chronic myeloid leukemia: A meta-analysis. Pharmacogenomics 15: 667-677, 2014.

24. Zheng Q, Wu H, Yu Q, Kim DH, Lipton JH, Angelini S, Soverini S, Vivona D, Takahashi N and Cao J: ABCB1 polymorphisms predict imatinib response in chronic myeloid leukemia patients: A systematic review and meta-analysis. Pharmacogenomics J 15: 127-134, 2015.

25. Lardo M, Castro M, Moiraghi B, Rojas F, Borda N, Rey JA and Lazarowski A: MDR1/ABCB1 gene polymorphisms in patients with chronic myeloid leukemia. Blood Res 50: 154-159, 2015.

26. Sung KW, Choi J, Hwang YK, Lee SJ, Kim HJ, Lee SH, Yoo KH, Jung HL and Koo HH: Overexpression of Apollon, an antiapoptotic protein, is associated with poor prognosis in childhood de novo acute myeloid leukemia. Clin Cancer Res 13: 5109-5114, 2007.

27. Ismail EA, Mahmoud HM, Tawfik LM, Habashy DM, Adly AA, El-Sherif NH and Abdelwahab MA: BIRC6/Apollon gene expression in childhood acute leukemia: Impact on therapeutic response and prognosis. Eur J Haematol 88: 118-127, 2012. 